\title{
Why is controlling blood pressure after stroke so difficult?
}

\author{
Martin Dawes MBBS MD
}

See related research article by Kerry and colleagues on page 23 and at www.cmaj.ca/lookup/doi/10.1503/cmaj.120832

I f you have a stroke and survive the first month, your likelihood of dying in the first year after the stroke is about $10 \%,{ }^{1}$ and your risk of having a second stroke in the next 10 years is $43 \%$. $^{2}$ Treatment of elevated blood pressure reduces the risk of recurrent stroke by $30 \% .^{3}$ Despite this major benefit, we as health professionals don't seem to be delivering that treatment adequately. ${ }^{1}$ According to the Public Health Agency of Canada, about 38000 Canadians have a stroke each year, with $80 \%$ surviving the acute event. ${ }^{4}$ The level of inadequate secondary prevention may be leading to an excess 1500 strokes and numerous deaths per year. The CMAJ article by Kerry and colleagues ${ }^{5}$ shows that, despite the most intensive efforts of multiprofessional teams, we don't seem to be able to control blood pressure adequately in large numbers of people who have survived stroke. Stroke is the second leading cause of death globally, ${ }^{6}$ which means this problem has major consequences for millions of people.

Elevated blood pressure is a risk factor for stroke. However, blood pressure is a physiologic variable that is difficult to measure accurately, is not symptomatic unless very high and is difficult to control. Sixty-seven years after the death of former US president Franklin D. Roosevelt from uncontrolled hypertension, we are still trying to deal with this problem. During that time, we have eradicated smallpox, nearly eradicated polio and discovered treatments for HIV/AIDS, and yet hypertension remains stubbornly difficult to control. The discovery of diuretics, calcium channel blockers, $\beta$-blockers, angiotensin-converting-enzyme inhibitors, angiotensin receptor blockers and $\alpha$-blockers as hypertension treatments have made control much more attainable.

Reasons for poor control of blood pressure involve both patients and health professionals. They include the individual response to antihypertensive medication, ${ }^{7}$ adverse effects of medication that may affect $20 \%$ of people, ${ }^{8}$ and the fact that only two-thirds of patients recently diagnosed with hypertension may be prescribed antihypertensive medication despite the use of clinical decision support systems. ${ }^{9}$ Health professionals' knowledge of practice guidelines ${ }^{10}$ may also be a factor. However, I believe that most health professionals know the evidence about the benefits of blood pressure control after stroke or transient ischemic attack, and the important lack of knowledge is probably about the specific benefit or harm the individual patient is likely to have. The epidemiologic evidence for benefit and risk is clear, but this does not mean that every patient gains benefit or that every patient sustains harm.

It may be slightly unusual to use a trial that did not show clear benefit of the intervention as a springboard for the promotion of personalized medicine. A physician has to make a diagnosis, evaluate the prognosis, identify therapeutic options and then share those with the patient. Making an accurate prognosis for a sick individual is difficult. The unknown elements of the prediction models include genomic and epigenetic information, and for most problems that information will not be available for a few years. This leads to some uncertainty when dealing with individual patients, which is the reason that guidelines are just guides.

There are no simple solutions, but for stroke survivors, the article by Kerry and colleagues ${ }^{5}$ shows there is a need for us to do more. The authors' trial was comprehensive and well done, in a population at high risk of recurrent stroke, and yet it did not show any benefit. We await the results of the Preventing Recurrent Vascular Events in Patients with Stroke or Transient Ischemic Attack (PREVENTION) study, the

\section{KEY POINTS}

- Stroke survivors are at high risk of recurrent stroke.

- Uncontrolled blood pressure is a major risk factor for recurrence of stroke.

- Controlling blood pressure is difficult to do in practice.

- Better awareness of this issue in primary care may be a start. 
only ongoing study in Canada that is addressing prevention of recurrent stroke in patients with hypertension.

We can adopt a very formulaic safety-check process for all survivors of stroke and transient ischemic attack, or we can carry on with the personalized approach that seems at the moment to be failing. Perhaps patients leaving the stroke unit could be given a prescriptive checklist to take to their primary care provider. Even this approach needs testing. Normally, I dislike the phrase "more research is needed" at the end of a research paper. However, when this many people are having a recurrent stroke, perhaps Canadians expect funding of more trials.

In the meantime, in the practice where I work, I will discuss with my colleagues how we might first identify all patients with a history of stroke through our electronic medical records and then run an audit on their blood pressure. I suspect we will identify a few patients with elevated blood pressure. Perhaps we could put alerts in their records to remind us at least of the risk associated with elevated blood pressure in these patients. We don't have a simple solution, but this will at least make us more aware.

\section{References}

1. Raine R, Wong W, Ambler G, et al. Sociodemographic variations in the contribution of secondary drug prevention to stroke survival at middle and older ages: cohort study. BMJ 2009;338:b1279.

2. Hardie K, Hankey GJ, Jamrozik K, et al. Ten-year risk of first recurrent stroke and disability after first-ever stroke in the Perth Community Stroke Study. Stroke 2004;35:731-5.

3. Lakhan SE, Sapko MT. Blood pressure lowering treatment for preventing stroke recurrence: a systematic review and metaanalysis. Int Arch Med 2009;2:30.

4. Tracking heart disease and stroke in Canada. Ottawa (ON): Public Health Agency of Canada; 2011. Available: www.phac-aspc .gc.ca/cd-mc/cvd-mcv/sh-fs-2011/pdf/StrokeHighlights_EN.pdf (accessed 2012 Nov. 7).

5. Kerry SM, Markus HS, Khong TK. Home blood pressure monitoring with nurse-led telephone support among patients with hypertension and a history of stroke: a community-based randomized controlled trial. CMAJ 2013;185:23-31.

6. Mathers CD, Boerma T, Ma Fat D. Global and regional causes of death. Br Med Bull 2009;92:7-32.

7. Dickerson JE, Hingorani AD, Ashby MJ, et al. Optimisation of antihypertensive treatment by crossover rotation of four major classes [see comments]. Lancet 1999;353:2008-13.

8. Bardage C, Isacson DG. Self-reported side-effects of antihypertensive drugs: an epidemiological study on prevalence and impact on health-state utility. Blood Press 2000;9:328-34.

9. Montgomery AA, Fahey T, Peters TJ, et al. Evaluation of computer based clinical decision support system and risk chart for management of hypertension in primary care: randomised controlled trial. BMJ 2000;320:686-90.

10. Hyman DJ, Pavlik VN. Self-reported hypertension treatment practices among primary care physicians: blood pressure thresholds, drug choices, and the role of guidelines and evidencebased medicine. Arch Intern Med 2000;160:2281-6.

Affiliation: Martin Dawes is with the Department of Family Practice, University of British Columbia, Vancouver, BC 